\title{
Peer review: Supporting reflective practice and connection between social workers
}

\author{
Anne Beets, Jolinda Bidois, Jill Broom, Paulette Moore, Lauren Swan
}

The authors are registered health social workers ${ }^{1}$ working for a District Health Board in a variety of services including surgical and medical services, community health services and mental health services. Collectively we have over 80 years of social work practice in statutory and non-governmental organisations.

\begin{abstract}
We describe the values, processes, shape and structure of a new social work peer review group. We identify some barriers to participating in peer review processes as well as highlighting the benefits. The value of regular practice review and development and the nurturing of connectedness are highlighted.
\end{abstract}

\section{Introduction}

When did you last have the opportunity to share your practice with peers? Does your work environment provide a formal regular opportunity to discuss social work practice issues? Clinical supervision is a support we all use regularly and find valuable, but recently we added in something new.

In our organisation, social workers are service based, managed by individual Clinical Nurse Managers or Service Managers. In former years there may have been more opportunities to be together as a group of social workers engaging in professional development and clinical discussion, but currently there is little opportunity for such interaction between social workers. Our organisational procedures require that all social workers engage in professional peer review (Lakes District Health Board, 2012). Peer review is defined as 'Processes which develop and maintain high standards of practice through constructive feedback from one social work professional to another with the desired outcome of clinical best practice' (Lakes District Health Board, 2012, p. 1). In our experience social work peer review has operated only intermittently over the last few years with different large group structures trialed and with varying amounts of enthusiasm over time.

A few of us wanted to do peer review in a different way to try and address what we perceived as barriers to a successful peer review format. The benefits to our practice have been great. We have used the process of writing this article to review and share our experience with other social workers.

1 One author has since moved to another role. 


\section{Getting started}

All it took to get started was two social workers meeting together and discussing how a regular peer review process could work. Initial literature searches led us to information about peer supervision. Peer review does have some characteristics similar to peer supervision. Peer supervision offers a safe environment to reflect on issues in practice and affirmation of strengths in practice (Polaschek, 2007). Peer review processes can also offer this opportunity.

Some aspects of group function and risks are present in both peer supervision and peer review. Writing about a leaderless peer supervision group, Hawkins and Shohet (2006) write of the importance of commitment from group members and clear structures to guide process as well as a way to pay attention to the group's own processes. These are also important in a leaderless peer review group such as ours, and are reflected in our terms of reference. McNicoll (2008) identifies a number of factors required for effective peer group supervision. Many of these also apply to the group processes involved in our peer review group including: importance of equality of being peers; having a supportive culture and a clear structure; prioritising attendance; and rules about confidentiality. For us, both supervision (of any format) and peer review are recognised as valid professional endeavours and we give them the same priority.

There are some differences we identify between peer supervision and peer review. Peer supervision features a supervision contract and clear, albeit rotating, roles for supervisor and supervisee (Hawkins \& Shohet, 2006; Polaschek, 2007). Polaschek (2007), writing about peer reciprocal supervision, highlights the need for a supervisee focus. In contrast our peer review group has terms of reference rather than a contract; does not use supervisor and supervisee roles nor aim to focus exclusively on the presenter of the day. We do not expect the same level of responsibility of one another that we would expect from a supervisor. We do expect that we will each take responsibility for ourselves, and that each person will maintain their own individual supervision. Peer review does not take the place of supervision, and cannot stand alone. We believe peer review is something different from peer supervision.

Invitations for expressions of interest to participate in a peer review group were sent out to all 28 social workers in our District Health Board. Following the initial expressions of interest the number of participants settled at five. Our organisational policy about social work peer review (Lakes District Health Board, 2012) gives some guidance in matters that should be considered in a peer review process. Together the five of us discussed, defined and agreed on our purpose, structure, parameters and commitment, creating our own terms of reference.

Within the peer review group the values of whakawhanaungatanga are in action. That is, being a group of people who support and strengthen our relationships and have developed an interconnectedness, similar to a kinship group. Manaakitanga guides us to take care of this kinship by maintaining openness and warmth to encourage a learning environment. Kaitiakitanga highlights the taking of responsibility both as individuals and as a group with awareness of what it is we each bring, presentz and own, and the limits of this within the group. Trust, respect, professionalism and commitment have been strong features of our group. 
A closed peer review group structure was chosen with the thought that maintaining a fixed membership could best foster commitment within the group as well as build trust. Commitment to attend was a requirement for membership. Issues a presenter could bring to the group included, but were not limited to, presentation of a case, information from training, conferences or articles, reflection on clinical themes that were common across cases, and celebration of typical daily work. Presentations did not need to be formal in style or follow any set format, but did need to be prepared and maintain a level of professionalism. Peer review discussions were confidential to the participants to enable open discussion and practice reflection. However, group members were welcome to take new learning, apply it to their own work and share it with others.

A process was agreed to address any concerns regarding a group member's practice that may arise within the peer review group. The process identified a preference of talking together as a group about the concerns, immediately where possible, seeking to reach consensus on action required, with the group taking supporting steps as needed. If this was not adequate to address the issues then a process would start which could include the clinician's supervisor.

Discussions were also held to identify potential and current dual roles present amongst our group members. Potential tensions and challenges that may arise from the complexity of these relationships were acknowledged. Agreement was reached that individuals would maintain themselves in a peer relationship during their participation in the group and that supervisory or other issues would be addressed outside the peer review group. Being aware, open and willing to discuss potential difficulties as they arose added to the success of the group.

An e-mail group and a shared access folder on the computer were set up for the exclusive use of the group. We used e-mail to share articles for reading, case studies, and group communications. We used the folder to save documents such as a roster of presenters and a record of attendance, with a brief summary of the topics reviewed. This record could be printed and used to provide evidence of this continuing practice development work for registration and for accountability to managers.

\section{The shape of our peer review group}

Our peer review group formed with five participants, a number small enough to ensure everyone had opportunity to contribute to discussions. Meetings of 45 minutes occurred on a weekly basis with a roster of presenters from the group. Each session began with a brief review of the previous week to provide a time to reflect on the previous topic and discuss any application of new learning. Often preparatory reading or a case study would be emailed to participants a day or two prior to the group.

Presentations would be followed with group discussion on the topic. Discussion included exchange of knowledge, affirmation and celebration of good practice, discussion of practice and theory, reflections on imperfect work, challenges to assumptions and practice. At sixmonth intervals we formally reviewed the peer review group process to consider whether we were continuing to achieve our goal of regular meetings that were beneficial for our practice, review group processes and consider any aspect that needed alteration. 
One member of the group was based in a rural service, and joined the peer review group by video link. We all felt uncertain about how well this could work, but experienced very few difficulties. Some initial training and familiarity with this arrangement helped us overcome any discomfort and technical ignorance. The risk of technology failures remained and did occur on a couple of occasions. The need to trust others in the group was highlighted, especially in trusting that confidentiality was being maintained at the other end. It was helpful to have video as well as audio capability so there was no loss of non-verbal communication, particularly helpful when discussing tough cases. Being able to participate in a peer review group was found to be helpful in reducing the isolation of working in a rural area. The ability to share and to discuss work in a caring professional group enhanced a sense of professional connection. Using video-conference facilities required good manners to ensure inclusive dialogue.

Some of the strength of our peer review group came from the diversity of the participants. We work and have worked in a range of different fields of practice and come from diverse cultures and backgrounds.

\section{The barriers}

Barriers to participating in a peer review may include:

- the pressures and ongoing stresses within the service workforce,

- reluctance to engage in a process that might be perceived as requiring more preparation than the benefits gained could justify,

- geographically isolated service,

- practice isolation,

- fear of exposing one's practice to others,

- workplace culture.

\section{The benefits}

We identified a number of areas of benefit for our practice and ourselves that resulted from participation in the peer review group. Some were expected and hoped for outcomes while others were unexpected but welcome benefits including that we:

- developed and grew in our practice,

- returned to our practice with fresh ideas and perspectives,

- celebrated examples of good practice,

- identified areas for improvement in our practice,

- exposed difficult or negative practice experience to supportive challenge,

- learned new things and were stimulated to learn more,

- had our sense of professional and personal belonging enhanced,

- felt nurtured as individuals and as social workers,

- raised awareness and the perceived value of professional development for other professions,

- built relationships and strengthened the interfaces between services,

- discussed points of tension across services. 


\section{Conclusion}

Peer review requires a willingness to disclose practice issues and to improve strength of practice knowledge by exposing areas of vulnerability or uncertainty as well as success. This requires a high degree of trust in the group sustaining a culture of supported learning. It has also required valuing our own practice enough to commit to the time allocated to review that practice. It is only by being willing to trial a different way of doing peer review that we experienced the benefits to fully overcome the above barriers.

We believe it is possible and beneficial for others to set up a peer review group, which may take a shape quite different from our own. We expect the shape of our own group to change and develop as time goes on, and as we consider how to include others in our own organisation. The shape of the group is perhaps less important than the values that guide the interactions within it.

Peer review quickly became something to look forward to. Our confidence has grown in presenting and exposing our practice to a group of peers. We have felt valued in our roles as social workers. Our knowledge has been expanded and reflective practice is supported. We are better social workers as a result of participating in regular peer review of our practice. A supportive learning culture can be created. The barriers can be overcome. Our peer review group has proven to provide a caring and enabling environment. It is recognised both by us and those we work with, and as an effective means of reviewing practice and enhancing connection among social workers.

\section{References}

Hawkins, P., \& Shohet, R. (2006). Supervision in the helping professions (3rd edition). Maidenhead: Open University Press.

Lakes District Health Board (2012). Peer review procedure for social work. social work management procedure document number 69225 (internal document). Rotorua: Lakes District Health Board.

McNicoll, A. (2008). Peer supervision - No-one knows as much as all of us: A background paper for workshop. Auckland: New Zealand Mentoring Centre. Retrieved August 24, 2012, from http://www.coachingmentoring.co.nz/resources / articles / 134-peer-supervision-no-one-knows-as-much-as-all-of-us.

Polaschek, L. (2007). Peer reciprocal supervision / whakaritenga tauutuutu kaitiakitanga. In D. Wepa (ed.), Clinical supervision in Aotearoa / New Zealand: A health perspective. (pp. 109-118). New Zealand: Pearson Education. 